\title{
Structural Evolution and Medium Range Order in Permanently Densified Vitreous $\mathrm{SiO}_{2}$
}

\author{
M. Zanatta, ${ }^{1,{ }^{*}}$ G. Baldi, ${ }^{2}$ R. S. Brusa, ${ }^{3}$ W. Egger, ${ }^{4}$ A. Fontana,${ }^{3}$ E. Gilioli, ${ }^{2}$ S. Mariazzi, ${ }^{3}$ G. Monaco, ${ }^{3}$ \\ L. Ravelli, ${ }^{4}$ and F. Sacchetti ${ }^{1,5}$ \\ ${ }^{1}$ Dipartimento di Fisica, Università di Perugia, I-06123 Perugia, Italy \\ ${ }^{2}$ IMEM-CNR, I-43124 Parma, Italy \\ ${ }^{3}$ Dipartimento di Fisica, Università di Trento, I-38123 Povo, Trento, Italy \\ ${ }^{4}$ Institut für Angewandte Physik und Messtechnik, Universität der Bundeswehr München, D-85577 Neubiberg, Germany \\ ${ }^{5}$ IOM-CNR clo Dipartimento di Fisica, Università di Perugia, I-06123 Perugia, Italy
}

(Received 2 August 2013; published 29 January 2014)

\begin{abstract}
Positron annihilation lifetime spectroscopy is employed to measure the size of the interstitial void spaces characterizing the structure of a set of permanently densified $\mathrm{SiO}_{2}$ glasses. The average volume of the voids is markedly affected by the densification process and linearly shrinks by almost an order of magnitude after a relative density variation of $22 \%$. In addition, $x$-ray diffraction shows that this change of density does not modify appreciably the short range order, which remains organized in $\mathrm{SiO}_{4}$ tetrahedra. These results strongly suggest a porous medium description for $v-\mathrm{SiO}_{2}$ glasses where the compressibility and the medium range order are dominated by the density variation of the voids volume up to densities close to that of $\alpha$-quartz.
\end{abstract}

DOI: 10.1103/PhysRevLett.112.045501

PACS numbers: 61.43.Fs, 71.60.+z, 78.70.Bj

Despite the absence of translational periodicity, the glassy structure is characterized by the presence of some degree of order on the short (SRO) and medium ranges (MRO) [1]. SRO is associated with well-defined first neighbors arrangements, which can be characterized in terms of bond lengths and bond angle distributions. The basic structural units composing the network of covalent glasses are coordination polyhedra packed in a nonrandom way to form a second level of organization, which typically extends over a length of a few interatomic distances $[2,3]$. The presence of MRO is revealed by the appearance of a first sharp diffraction peak (FSDP) in the static structure factor $S(Q)$. The FSDP has been observed in a wide range of glasses, supercooled liquids, and even in melts at high temperature, displaying a universal dependence on temperature and pressure [4]. This suggests a common structural origin, associated to the atomic arrangement at the nanometer scale. Several interpretation schemes have been proposed to explain this feature. The layered structure of many glass-forming chalcogenide glasses has suggested the identification of the FSDP with a Bragg-like peak originated by pseudocrystalline arrangements [5]. On the other hand, the FSDP has been connected to the occurrence of characteristic low density regions [6,7]. These void structures are surrounded by chemically ordered clusters leading to correlation distances typical of the MRO [4]. Thus, the FSDP arises from a prepeak in the concentration-concentration structure factor in the Bhatia-Thornton formalism [8].

The importance of the nanoscale structure in the glass transition phenomenology has been pointed out in recent numerical works highlighting some connection between heterogeneous dynamics [9] and the structural order
$[10,11]$. The nanometer length scale is also relevant in the glassy state below the glass transition temperature, where it marks the transition from a macroscopic elastic regime to a microscopic one where the dynamics of the glass and that of the corresponding density polycrystal become very similar [12]. However, the knowledge of the real-space arrangement at the MRO scale is still elusive and practically accessible only in computer simulations. In fact, Fourier transform of the $S(Q)$ only produces subtle MRO correlations, whereas the effectiveness of imaging techniques at the nanometer scale is still currently limited.

In this Letter, we present a study of the density evolution of the structure of vitreous silica $v-\mathrm{SiO}_{2}$, archetype of covalent network-forming glasses and one of the most investigated case studies, e.g., [13]. The structural building blocks are rather well defined $\mathrm{SiO}_{4}$ tetrahedra arranged in an open network similar to that of the high-temperature crystalline $\mathrm{SiO}_{2}$ polymorph $\beta$-cristobalite [14]. A highpressure high-temperature permanent densification procedure was exploited to produce samples up to a $22 \%$ of densification, close to the $\alpha$-quartz density [15]. The evolution with density of the structural voids has been probed by means of positron annihilation lifetime spectroscopy (PALS), and the SRO has been characterized by means of x-ray diffraction (XRD). We find that, up to the density of $\alpha$-quartz, the $v-\mathrm{SiO}_{2}$ structure can be described in terms of that of a porous medium, with an incompressible and a compressible component. The former one corresponds to the $\mathrm{SiO}_{4}$ tetrahedra whereas the latter one is related to the interstitial voids, whose volume shrinks on increasing density and is reduced to $\sim 3 \%$ of its ambient pressure value at densities close to the $\alpha$-quartz one. The 
observed density variation of the interstitial voids dominates the correlations originating the MRO and well accounts for the observed density dependence of the FSDP, consistent with the void-cluster picture for the glassy network $[6,7]$.

Permanently densified silica samples were prepared starting from the same commercial-grade Spectrosil block purchased from SILO (Florence, Italy). The material was drilled in cylindrical pieces of about $4 \mathrm{~mm}$ diameter and $4 \mathrm{~mm}$ length and then loaded into a 6/8-type multianvil apparatus (Rockland Research Corporation). The pressure was first increased at a rate of $1 \mathrm{GPa}$ /hour up to the final value, and the temperature was then raised to $770 \mathrm{~K}$ with a heating rate of $50 \mathrm{~K} / \mathrm{min}$ and kept there for 10 minutes. The sample was finally quenched and the pressure was released at a rate of $0.3 \mathrm{GPa} /$ hour. The final products are homogeneous and densified glasses. The density of the obtained samples has been found to be stable over at least a few years, i.e., over a time scale much longer than the one corresponding to the investigations discussed here. The applied pressures and the corresponding densities and densification ratios are reported in Table I. In the following, we refer to the samples using their densification pressures.

PALS experiments were performed at the pulsed low energy positron system (PLEPS) [16], which is coupled to the neutron induced positron source Munich (NEPOMUC) [17], located at the research reactor FRMII (Garching, Germany). In a PALS experiment, the main observable is the time elapsing between the positron $e^{+}$implantation and its annihilation. Spectra were acquired at two implantation energies $E_{i}$ of 16 and $18 \mathrm{keV}$ by accumulating several million annihilation events. The average implantation depth $\bar{z}\left(E_{i}, \rho\right)$ ranges between 1 and $2 \mu \mathrm{m}$ [18]; however, the measured spectra do not show any $E_{i}$ dependence, thus, confirming the sample homogeneity. Figure 1(a) shows PALS spectra measured at $E_{i}=16 \mathrm{keV}$ as a function of the increasing density. The spectral shape is given by the convolution between the sum of $N$ exponential decays $z_{i}(t)$ and the experimental time resolution function $R(t)$ [19] superimposed onto a constant background $b k g$, namely:

$$
Z(t)=R(t) \otimes \sum_{i=1}^{N} \frac{I_{i}}{\tau_{i}} \exp \left(-t / \tau_{i}\right)+b k g,
$$

where $\tau_{i}$ and $I_{i}$ are the lifetime and intensity of the different annihilation contributions, respectively. In insulators with open volumes, i.e., a region with low electron density, $e^{+}$ can also form positronium (Ps), an $e^{+}-e^{-}$bound state. This process is very effective in $v-\mathrm{SiO}_{2}$ where up to $80 \%$ of the implanted $e^{+}$forms positronium [20]. Ps exists in two spin states: the singlet state parapositronium (pPs, lifetime in vacuum $\tau_{p}=125 \mathrm{ps}$ ) and the long living triplet state orthopositronium (oPs, lifetime in vacuum $\tau_{o}=142 \mathrm{~ns}$ ). In condensed matter, the latter lifetime is reduced via pickoff annihilation with the electron cloud limiting the void volume. However, the pickoff lifetime is still longer than that of the other annihilation processes and it conveys information on the void dimensions.

The measured spectra were analyzed by means of the PATFIT package [21]. An example of spectral deconvolution is shown in Fig. 1(b). The analysis points out the presence of three lifetime components: (i) a short lifetime $\tau_{1}$ which comes from $\mathrm{pPs}$ and free positron annihilation in bulk silica; (ii) an intermediate lifetime $\tau_{2}$ ranging from 733 ps, corresponding to the oPs pickoff annihilation in small voids, to $359 \mathrm{ps}$, close to the characteristic time ( $\sim 300 \mathrm{ps}$ ) of positron annihilation in $\mathrm{Si}$ or $\mathrm{O}$ vacant sites in crystalline $\mathrm{SiO}_{2}$ [22]; (iii) a long lifetime $\tau_{3}$ that is related to oPs pickoff in the intrinsic voids of the amorphous structure. The decrease of this longest decaying lifetime going from normal silica to the $8 \mathrm{GPa}$ sample is evident from Fig. 1(a) and represents the main outcome of the present PALS experiment. In the most densified sample, it is necessary to add a further long-living component with a lifetime $\tau_{4} \sim 3 \mathrm{~ns}$ to accurately describe the spectrum. The intensity $I_{4}$, possibly related to oPs pickoff in small cracks, is less than $1.5 \%$ of the total intensity and, thus, marginal. The $E_{i}$-averaged values of the lifetimes and their corresponding intensities are reported in Table I.

On increasing the density, both the lifetime $\tau_{3}$ and the corresponding intensity $I_{3}$ decrease, see the inset in Fig. 2. The decrease of $\tau_{3}$ is related to the shrinking of the intrinsic voids in the silica samples, while $I_{3}$ indicates a reduction of the oPs formation and, consequently, of the number of intrinsic voids. At the same time the lifetime $\tau_{2}$ also decreases while the corresponding intensity $I_{2}$ increases, see inset in Fig. 2. It is worth noting that the strong decrease in the Ps formation on increasing the sample density leads also to the decrease in the fraction of pPs self annihilating

TABLE I. Applied pressure, density, and densification value for the probed samples. The density was measured by the Archimedes method using ethanol as immersion fluid. $E_{i}$-averaged lifetimes $\tau_{i}$ and intensities $I_{i}$ are also reported.

\begin{tabular}{llllccccccc}
\hline \hline Sample & $\begin{array}{c}\text { Density } \\
\left(\mathrm{g} / \mathrm{cm}^{3}\right)\end{array}$ & $\begin{array}{c}\text { Densification } \\
(\%)\end{array}$ & $\begin{array}{c}\tau_{1} \\
(\mathrm{ps})\end{array}$ & $\begin{array}{c}\tau_{2} \\
(\mathrm{ps})\end{array}$ & $\begin{array}{c}\tau_{3} \\
(\mathrm{ps})\end{array}$ & $\begin{array}{c}\tau_{4} \\
(\mathrm{ps})\end{array}$ & $\begin{array}{c}I_{1} \\
(\%)\end{array}$ & $\begin{array}{c}I_{2} \\
(\%)\end{array}$ & $\begin{array}{c}I_{3} \\
(\%)\end{array}$ & $\begin{array}{c}I_{4} \\
(\%)\end{array}$ \\
\hline $0 \mathrm{GPa}$ & $2.198(5)$ & 0 & $145 \pm 3$ & $730 \pm 30$ & $1623 \pm 6$ & & $25.5 \pm 0.5$ & $18.7 \pm 0.5$ & $55.8 \pm 0.6$ & \\
$4 \mathrm{GPa}$ & $2.255(5)$ & 2.6 & $165 \pm 3$ & $571 \pm 10$ & $1540 \pm 20$ & & $26.4 \pm 0.3$ & $28.9 \pm 1.5$ & $47.7 \pm 1.7$ & \\
$6 \mathrm{GPa}$ & $2.406(5)$ & 9.5 & $195 \pm 12$ & $570 \pm 50$ & $1250 \pm 20$ & & $29.3 \pm 0.3$ & $40.9 \pm 0.7$ & $29.8 \pm 2.0$ & \\
$8 \mathrm{GPa}$ & $2.67(1)$ & 22 & $169 \pm 6$ & $359 \pm 5$ & $781 \pm 14$ & $3590 \pm 80$ & $39.7 \pm 4.0$ & $45.3 \pm 4.0$ & $13.9 \pm 0.2$ & $1.1 \pm 0.1$ \\
\hline \hline
\end{tabular}



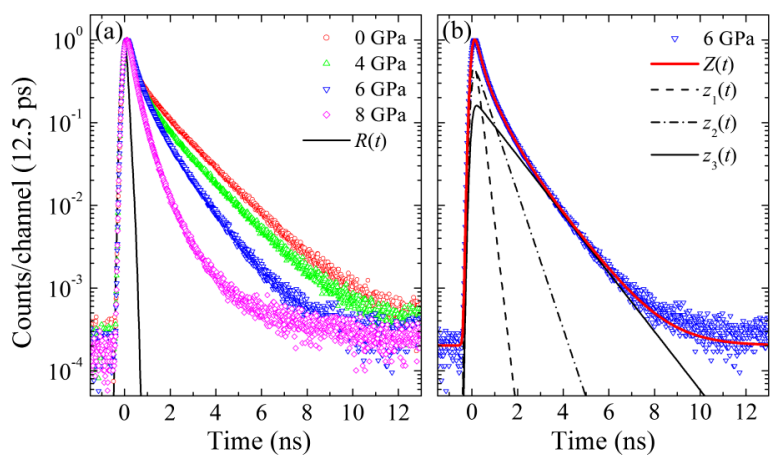

FIG. 1 (color online). (a) Lifetime spectra measured at $16 \mathrm{keV}$ positron implantation energy in $v-\mathrm{SiO}_{2}$ samples with increasing density (from top to bottom). Spectra are normalized to the peak height. The instrumental time resolution function $R(t)$ is also reported (solid black line), FWHM $=261.9$ ps. (b) Example of spectral deconvolution for the $6 \mathrm{GPa}$ sample signal. The thick red line is the best fit to the data whereas black lines represent the three lifetime components.

with the characteristic $125 \mathrm{ps}$ lifetime, and then the increase of the lifetime $\tau_{1}$.

The lifetime $\tau_{3}$, measuring the oPs pickoff annihilation, can be related to the interstitial voids dimension by the TaoEldrup model $[23,24]$. The model assumes a spherical void with an effective radius $R$ described by an infinite potential well in which Ps is trapped in the ground state and annihilates with the electrons only when its wave function penetrates into the bulk. These annihilations are taken into account by an empirical parameter $\Delta R$ : orthopositronium is assumed to have an infinite lifetime inside the potential well of radius $R$, whereas the spin-averaged Ps lifetime within the penetration distance $\Delta R$ from the walls of the potential well is $0.5 \mathrm{~ns}$. The model is adequate for $R<12.5 \AA$. The penetration distance $\Delta R$ in silica was calibrated to be $1.68 \AA$ [25]. The density evolution of the

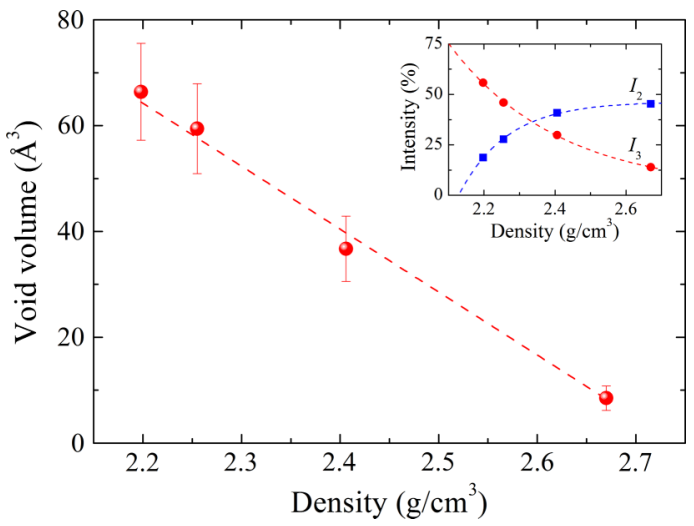

FIG. 2 (color online). Interstitial void volume $\frac{4}{3} \pi R^{3}$ as obtained from PALS data. The dashed line is a linear fit to the data. The density-evolution of the intensities $I_{2}$ (blue squares) and $I_{3}$ (red circles) is reported in the inset; the dashed lines are guides for the eyes. The oPs formation in normal vitreous silica is about $56 \%$ of the implanted positrons, while another $18 \%$ forms pPs. volume of the voids is reported in Fig. 2; increasing the density by $22 \%$ the voids volume linearly shrinks to $12 \%$ of its initial value.

Additional information on the evolution of the short range structure as well as of the FSDP was obtained by means of x-ray diffraction. The measurements were performed on a two axis diffractometer using the $\mathrm{Ag} \mathrm{K}_{\alpha}$ radiation $(\lambda=0.5608 \AA)$ monochromatized by a graphite crystal. Figure 3(a) shows the static structure factor $S(Q)$ for normal $v$ - $\mathrm{SiO}_{2}$ and the permanently densified silica samples. The high- $Q$ part of $S(Q)$ is almost unchanged, showing that the short range structure, i.e., the $\mathrm{SiO}_{4}$ tetrahedra, are scarcely affected by densification, as already shown in Refs. [26,27]. This is also clearly visible by computing the number density function $N(r)$ [28]. This quantity is reported in Fig. 3(b) and gives information on the average number of atoms at a given distance $r$. At distances smaller than the oxygen-oxygen distance $r_{\mathrm{OO}}$ $N(r)$ is almost unchanged, whereas it increases at the intertetrahedra length scale. On the contrary, the densification process induces a modification in the MRO range as indicated by the FSDP, see Fig. 3(c). In fact, on increasing density the FSDP position $Q_{1}$ shifts upwards by about $20 \%$ while its width $\Delta Q_{1}$ remains constant. It is worth noting that the position $Q_{1}$ of the FSDP at $4 \mathrm{GPa}$ basically corresponds to that of the most intense Bragg peak of $\alpha$ cristobalite and that of the $8 \mathrm{GPa}$ sample to that of $\alpha$-quartz, see Fig. 3(c).

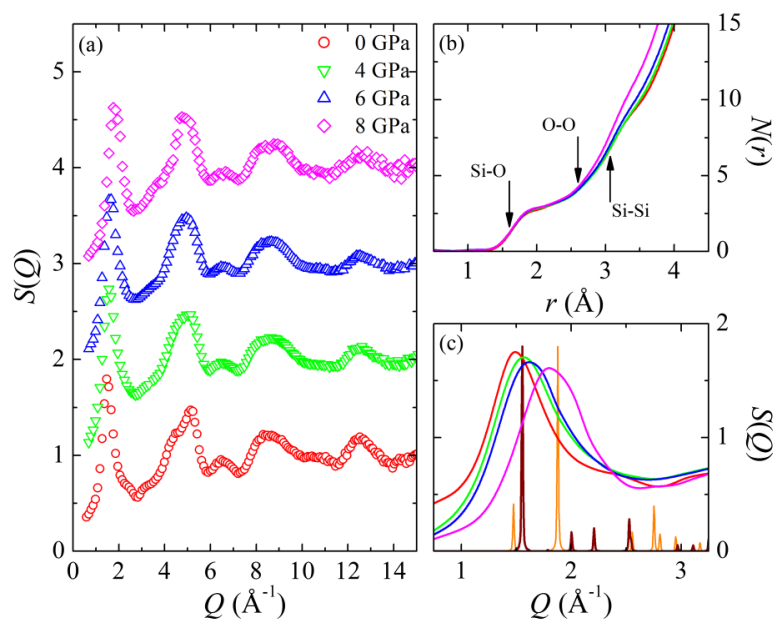

FIG. 3 (color online). (a) Static structure factor for normal silica and permanently densified samples. (b) Density evolution of the number distribution function $N(r)$; density increases from bottom to top [colors are as in the legend of (a)]. The bond lengths are marked by black arrows: $r_{\mathrm{SiO}}=1.60 \AA, r_{\mathrm{OO}}=2.5 \AA$, and $r_{\mathrm{SiSi}}=3.07 \AA[13,26]$. (c) Comparison between the low- $Q$ portion of the diffraction patterns of the permanently densified $-v-\mathrm{SiO}_{2}$ samples [density increases from left to right; colors as in the legend of (a)] and of the crystalline $\mathrm{SiO}_{2}$ polymorphs $\alpha$-quartz (orange solid line) and $\alpha$-cristobalite (brown thick solid line). 


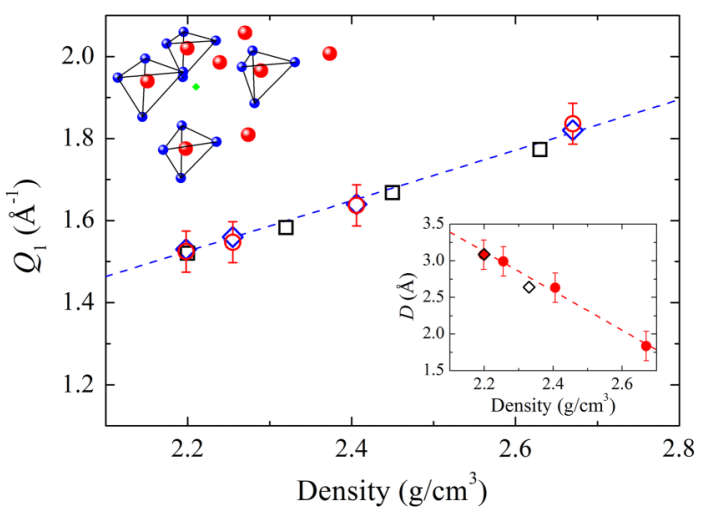

FIG. 4 (color online). FSDP position $Q_{1}$ as obtained by means of XRD (blue open diamonds) and neutron diffraction from Ref. [26] (black open squares) compared to the positions calculated using the void-cluster model (red open circles). (a) Structure of ideal $\beta$-cristobalite [33], silicon, and oxygen atoms are represented as big (red) and small (blue) dots, respectively; the green diamond represents the center of the void space. (b) Density dependence of the Si-void center distance $D$ for permanently densified $v-\mathrm{SiO}_{2}$ (red circles) and the geometrical distance $D$ calculated for $\alpha$ and $\beta$-cristobalite (black diamonds).

The XRD and PALS results naturally suggest a description of the $v-\mathrm{SiO}_{2}$ structure using a porous medium model, with an incompressible and a compressible part. The compressible part can be estimated from the voids volume fraction $f_{v}=\mathrm{CI}_{3} v$, where $v$ is the average volume of a single void, $I_{3}$ is proportional to the concentration of voids and $C$ is a material-dependent constant [29,30]. Combining the measured values of $I_{3}$ and $v$ (see Fig. 2), we obtain that a $22 \%$ increase in density gives rise to a shrinking of the compressible component to $\sim 3 \%$ of its initial value. This implies that the compressible part of the structure of ambient pressure $v$ - $\mathrm{SiO}_{2}$, i.e., the interstitial voids, occupy $\sim 18 \%$ of the total volume. The reduction of the compressible part upon densification can be ascribed to an almost rigid rotation of the tetrahedra which fill the interstitial voids [31]. Only at pressures corresponding to densities larger than that of $\alpha$-quartz the short range arrangement will be affected, and in fact, at that point, the $\mathrm{Si}$ coordination starts to increase from fourfold to sixfold [32].

Moreover, the FSDP variation with density can also be explained in terms of reduction of the voids volume. The glassy network can be imagined as an ensemble of voids surrounded, at a distance $D$ from their centers, by quasispherical cation centered clusters. For vitreous silica, these clusters are $\mathrm{SiO}_{4}$ tetrahedra separated by the $\mathrm{Si}-\mathrm{Si}$ distance $d$. The FSDP is, thus, due to the chemical ordering of the clusters around the void and is related to a prepeak in the concentration-concentration partial structure factor $S_{\mathrm{CC}}(Q)$ in the Bhatia-Thornton formalism [8]. The peak position $Q_{1}$ turns out to be [7]

$$
Q_{1}^{t h}=\frac{3 \pi}{2 d}\left(\frac{3}{2}-\frac{D}{2 d}\right)
$$

where $d=r_{\mathrm{SiSi}}=3.07 \AA$ and $D$ is estimated by the PALS data in the following way. The Si-void center distance can be obtained as $D=R_{\text {void }}+r_{\text {eff }}$, where $r_{\text {eff }}$ is a screening distance accounting for the exact location of the high electron-density region which cannot be explored by positrons. To estimate $r_{\text {eff }}$ we can compare the value of $R$ measured in $v-\mathrm{SiO}_{2}$ at ambient density to the geometrical $\mathrm{Si}$-void center distance calculated in a $\beta$-cristobalite idealcell (density $2.20 \mathrm{~g} / \mathrm{cm}^{2}$ ) [33] reported in Fig. 4(a). We obtain that $r_{\text {eff }} \sim 0.59 \AA$. Using this value, we can calculate the density dependence of $D$ as reported in Fig. 4(b). Using these values, finally, we can compute via Eq. (2) the values of $Q_{1}^{\text {th }}$ shown in Fig. 4 together with the measured ones. It is clear that the present PALS data allow us to describe with great accuracy both the FSDP position and its density dependence in absolute units within the void-cluster model [7].

In conclusion, XRD measurements in permanently densified $v-\mathrm{SiO}_{2}$ show that a density increase of up to $22 \%$ does not modify appreciably the short range structure, which remains organized in rigid $\mathrm{SiO}_{4}$ tetrahedra. On the contrary PALS results allow us to study the density dependence of the interstitial voids size. The structure of $v-\mathrm{SiO}_{2}$ comes up to be that of a porous medium, with pores that in $v-\mathrm{SiO}_{2}$ at ambient conditions have a radius $R=$ $2.5 \pm 0.2 \AA$ and occupy $\sim 18 \%$ of the total volume, and that display linear decrease of their volume with increasing density up to the density of $\alpha$-quartz. The reduction of the interstitial void space dominates the variation of the medium range correlations and finally the $v-\mathrm{SiO}_{2}$ compressibility; it also accurately describes the density dependence of the FSDP position via the void-cluster model $[6,7]$.

The Forschungs-Neutronenquelle Heinz Maier-Leibnitz (FRMII) facility is gratefully acknowledged for allocation of beam time at the NEPOMUC facility.

*marco.zanatta@fisica.unipg.it

[1] S. R. Elliott, Nature (London) 354, 445 (1991).

[2] J. D. Martin, S. J. Goettler, N. Fosse, and L. Iton, Nature (London) 419, 381 (2002).

[3] P. S. Salmon, R. A. Martin, P. E. Mason, and G. J. Cuello, Nature (London) 435, 75 (2005).

[4] S. R. Elliott, J. Phys. Condens. Matter 4, 7661 (1992).

[5] P. H. Gaskell and D. J. Wallis, Phys. Rev. Lett. 76, 66 (1996).

[6] J. Blétry, Philos. Mag. B 62, 469 (1990).

[7] S. R. Elliott, Phys. Rev. Lett. 67, 711 (1991).

[8] A. B. Bhatia and D. E. Thornton, Phys. Rev. B 2, 3004 (1970).

[9] L. Berthier and G. Biroli, Rev. Mod. Phys. 83, 587 (2011).

[10] T. Kawasaki, T. Araki, and H. Tanaka, Phys. Rev. Lett. 99, 215701 (2007). 
[11] H. Tanaka, T. Kawasaki, H. Shintani, and K. Watanabe, Nat. Mater. 9, 324 (2010).

[12] G. Baldi, M. Zanatta, E. Gilioli, V. Milman, K. Refson, B. Wehinger, B. Winkler, A. Fontana, and G. Monaco, Phys. Rev. Lett. 110, 185503 (2013).

[13] A. C. Wright, J. Non-Cryst. Solids 179, 84 (1994).

[14] J. P. Rino, I. Ebbsjö, R. K. Kalia, A. Nakano, and P. Vashishta, Phys. Rev. B 47, 3053 (1993).

[15] M. Zanatta, G. Baldi, S. Caponi, A. Fontana, E. Gilioli, M. Krish, C. Masciovecchio, G. Monaco, L. Orsingher, F. Rossi, G. Ruocco, and R. Verbeni, Phys. Rev. B 81, 212201 (2010).

[16] W. Egger, in Physics with Many Positrons, edited by R. S. Brusa, A. Dupasquier, and A. P. Mills, Jr. (North Holland, Amsterdam, 2010), p. 419.

[17] C. Hugenschmidt, in Physics with Many Positrons, edited by R. S. Brusa, A. Dupasquier, and A. P. Mills, Jr. (North Holland, Amsterdam, 2010), p. 399.

[18] P. Asoka-Kumar, K. G. Lynn, and D. O. Welch, J. Appl. Phys. 76, 4935 (1994).

[19] The resolution function $R(t)$ at each positron implantation energy is obtained by measuring a $p$-type silicon carbide reference sample which is characterized by a bulk lifetime of $145 \mathrm{ps}$ and a surface lifetime of 386 ps. Fixing the two lifetimes and the background, the measured signal is deconvolved to obtain $R(t)$; see also, W. Egger, G. Kögel, P. Sperr, and H. J. Gudlat, Int. J. Mat. Res. 97, 1633 (2006).

[20] P. Mazzoldi, G. Mattei, L. Ravelli, W. Egger, S. Mariazzi, and R. S. Brusa, J. Phys. D 42, 115418 (2009).

[21] P. Kirkegaard, N. J. Pedersen, and M. Eldrup, PATFIT, RISO National Laboratory, Denmark, 1998.
[22] M. Hasegawa, M. Saneyasu, M. Tabata, Z. Tang, Y. Nagai, T. Chiba, and Y. Ito, Nucl. Instrum. Methods Phys. Res., Sect. B 166-167, 431 (2000).

[23] S. J. Tao, J. Chem. Phys. 56, 5499 (1972).

[24] M. Eldrup, D. Lightbody, and J. N. Sherwood, Chem. Phys. 63, 51 (1981).

[25] D. W. Gidley, W. E. Frieze, T. L. Dull, A. F. Yee, E. T. Ryan, and H. M. Ho, Phys. Rev. B 60, R5157 (1999).

[26] Y. Inamura, M. Arai, T. Otomo, N. Kitamura, and U. Buchenau, Physica (Amsterdam) 284-288, 1157 (2000).

[27] S. Susman, K. J. Volin, D. L. Price, M. Grimsditch, J. P. Rino, R. K. Kalia, P. Vashishta, G. Gwanmesia, Y. Wang, and R. C. Liebermann, Phys. Rev. B 43, 1194 (1991).

[28] The number density function $N(r)$ is defined as: $N(r)=4 / 3 \pi r^{3} n f_{w}+2 / \pi \int_{0}^{Q_{m}} \mathrm{~d} Q Q[S(Q)-1](\sin (r Q)-$ $r Q \cos (r Q)) / Q^{2}$ where $f_{w}=\left(\sum_{i} Z_{i}\right)^{2} / \sum_{i} Z_{i}^{2}, n$ is the molecular density, and $Z_{i}$ is the nuclear charge of the $i$ th atom.

[29] Y. Kobayashi, W. Zheng, E. F. Meyer, J. D. McGervey, A. M. Jamieson, and R. Simha, Macromolecules 22, 2302 (1989).

[30] P. E. Mallon in Principles and Applications of Positron and Positronium Chemistry, edited by Y. C. Jean, P. E. Mallon, and D. M. Schrader (World Scientific, Singapore, 2003), p. 253.

[31] S. Sampath, C. J. Benmore, K. M. Lantzky, J. Neuefeind, K. Leinenweber, D. L. Price, and J. L. Yarger, Phys. Rev. Lett. 90, 115502 (2003).

[32] S. Sugai and A. Onodera, Phys. Rev. Lett. 77, 4210 (1996).

[33] W. W. Schmahl, I. P. Swainson, M. T. Dove, and A. GraemeBarber, Z. Kristallogr. 201, 125 (1992). 